\title{
Nitroglycerin use in obstetrical anesthesia: a multicentre survey of Canadian anesthesiologists
}

\author{
Thomas Drew, MD · Gayani Jayasooriya, MD · Jose C. A. Carvalho, MD, PhD • \\ Mrinalini Balki, MD, MBBS
}

Received: 30 January 2020/Revised: 26 February 2020/ Accepted: 26 February 2020/Published online: 30 March 2020

(c) Canadian Anesthesiologists' Society 2020

\section{To the Editor,}

Nitroglycerin is a potent tocolytic agent frequently used to achieve rapid uterine relaxation during obstetric emergencies. ${ }^{1}$ The use of nitroglycerin in Canadian obstetrical anesthesia practice is unknown and no published guidelines exist for its indications or administration in Canada (or elsewhere). We sought to investigate the current practice across Canadian Anesthesiologists' Society (CAS) members through the dissemination of a web-based nationwide survey.

After institutional research ethics board and CAS executive committee approval, a link to the survey at typeform.com was distributed to 2,334 CAS members with two follow-up reminder emails one week apart. All responses were anonymous and consent was implied by participation in the survey. The survey instrument was designed to assess indications, dose, administration route, and the effect of nitroglycerin administration on uterotonic drug dosing. Content was constructed through literature review, item reduction, and response formatting. ${ }^{2}$ The survey was tested using a convenience sample of four anesthesiologists with their suggestions incorporated into the final version. Non-probability purposive sampling was used as it was unknown what proportion of CAS members had an obstetric anesthesia component to their clinical

Electronic supplementary material The online version of this article (https://doi.org/10.1007/s12630-020-01614-7) contains supplementary material, which is available to authorized users.

T. Drew, MD ( $\varangle) \cdot$ G. Jayasooriya, MD .

J. C. A. Carvalho, MD, PhD - M. Balki, MD, MBBS

Department of Anesthesiology and Pain Management, Mount

Sinai Hospital, University of Toronto, Toronto, ON, Canada

e-mail: drthomasdrew@gmail.com practice. An initial question identified respondents who did not provide any obstetric anesthesia services and these respondents were excluded from answering further questions. There was no minimum threshold of provided obstetric anesthesia services for participation. The survey contained initial demographic questions followed by a question about obstetric indications for nitroglycerin administration (Figure). Participants were then informed that the following dose questions should be considered in the clinical context of difficult fetal extraction at Cesarean delivery. Questions were of binary, multiple choice, and free-text style.

Of the 2,334 contacted, 175 (8\%) completed the survey representing 102 centres across all 11 Canadian provinces/ territories. The survey questions and results are contained in the eAppendix (available as Electronic Supplementary Material). The Figure shows the obstetric clinical indications that respondents considered for nitroglycerin use. The intravenous formulation was preferred by $139 / 175$ (79\%) compared with $36 / 175$ (21\%) using the sublingual metered spray. There was wide variability in the intravenous nitroglycerin dose administered for rapid uterine relaxation with 74/175 (42\%) using an initial dose of $50 \mu \mathrm{g}, 55 / 175(31 \%)$ using $100 \mu \mathrm{g}$, and 32/175 (18\%) using $200 \mu \mathrm{g}$. Four percent $(7 / 175)$ reported that they would never consider intravenous administration. In those patients with oxytocin exposure during labour, 104/175 (59\%) of respondents would not alter the nitroglycerin dose, whereas 15/175 (9\%) would increase and 4/175 (2\%) would decrease the dose; 52/175 (30\%) were unsure if they would increase or decrease the dose.

With regards to oxytocin dosing during the third stage of labour after the administration of predelivery nitroglycerin for rapid uterine relaxation, 103/175 (59\%) of respondents would administer the same oxytocin dose, 32/175 (18\%) 
Figure Responses to the survey question, "In which obstetric situations would you consider using nitroglycerin?"

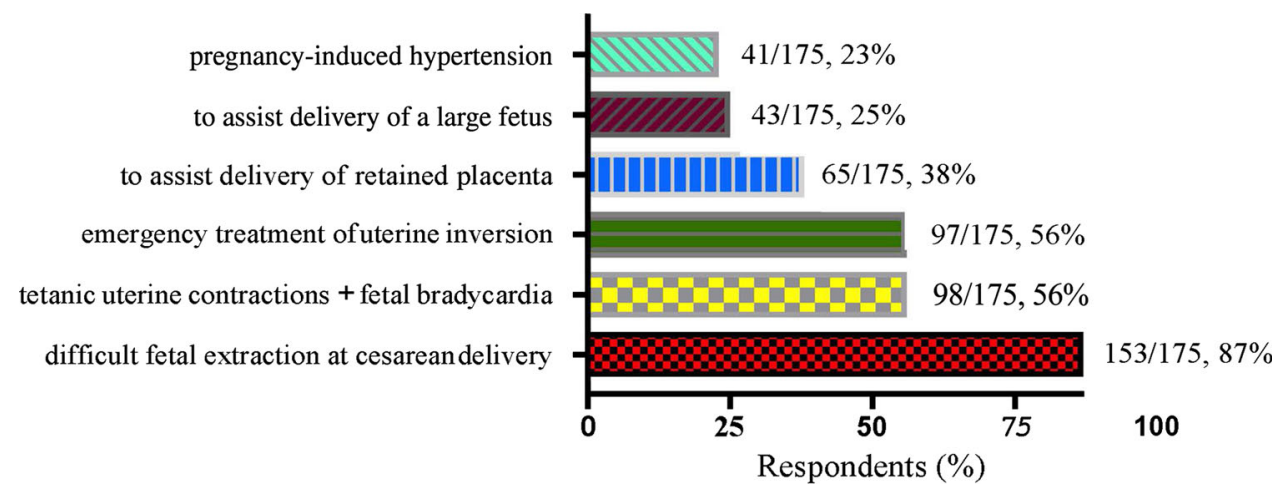

would choose a higher dose, 8/175 (5\%) would use a lower dose, and 32/175 (18\%) were unsure. Fifty-five percent (96/175) of respondents would not expect the patient to require more than one postpartum uterotonic agent, and 42/ 175 (24\%) of respondents expected a second uterotonic drug would be required; 37/175 (21\%) were unsure. Respondents based their current practice of nitroglycerin usage on perceived effectiveness (110/175, 63\%), availability $(107 / 175,61 \%)$, and individual preference $(100 / 175,57 \%)$ as opposed to local departmental protocols $(36 / 175,21 \%)$, scientific literature, $(24 / 175,14 \%)$ or society guidelines $(19 / 175,11 \%)$.

The survey results were limited by the very low response rate, leading to a high risk of sampling bias, which may affect the external validity of these results. Lack of time and increased survey burden are often proposed to contribute to low physician response rates in web-based surveys. ${ }^{3}$

Our results reveal substantial variability in nitroglycerin use by Canadian anesthesiologists in obstetrics. Lack of guidelines and evidence supporting the use of nitroglycerin may contribute to this variability. Further research into the use of nitroglycerin for obstetric indications is required to fill these knowledge gaps.

\section{Conflicts of interest None.}

Funding statement Dr. Mrinalini Balki and Dr. Jose Carvalho are supported by a Merit Award from the Department of Anesthesia, University of Toronto.

Editorial responsibility This submission was handled by Dr. Hilary P. Grocott, Editor-in-Chief, Canadian Journal of Anesthesia.

\section{References}

1. Morgan PJ, Kung R, Tarshis $J$. Nitroglycerin as a uterine relaxant: a systematic review. J Obstet Gynaecol Can 2002; 24: 403-9.

2. Burns KE, Duffett $M$, Kho $M E$, et al. A guide for the design and conduct of selfadministered surveys of clinicians. CMAJ 2008; 179: 245-52.

3. Cunningham CT, Quan H, Hemmelgarn B, et al. Exploring physician specialist response rates to web-based surveys. BMC

Publisher's Note Springer Nature remains neutral with regard to jurisdictional claims in published maps and institutional affiliations. Med Res Methodol 2015; 15: 32 